\section{Palofra}

acceso $\partial$ abierto

\title{
Los colectivos de las víctimas en aspectos sociodemográficos, formación, trayectorias laborales y expectativas de inserción laboral: revisión bibliográfica
}

\author{
Victims' collectives in sociodemographic aspects, training, \\ laboral trajectories and labor insertion expectations: \\ bibliographic review
}

Cómo citar: Cárdenas, S., Anacona, J., \& Millán, E. (2021). Los colectivos de las víctimas en aspectos sociodemográficos, formación, trayectorias laborales y expectativas de inserción laboral: revisión bibliográfica. Palobra, 21(1), 61-79. https://doi.org/10.32997/2346-2884-vol.21num.1-2021-3487

Recibido: 30 de agosto de 2020 .

Aprobado: 02 de marzo de 2021.

Editor: Ricardo Chica Gelis. Universidad de Cartagena-Colombia.

Tipología IBN Publindex:

Artículo de Revisión.

Copyright: (๑) 2021. Cárdenas, S., Anacona, J., \& Millán, E. Este es un artículo de acceso abierto, distribuido bajo los términos de la licencia https://creativecommons.org/licenses/bync-sa/4.0/ la cual permite el uso sin restricciones, distribución y reproducción en cualquier medio, siempre y cuando que el original, el autor y la fuente sean acreditados.

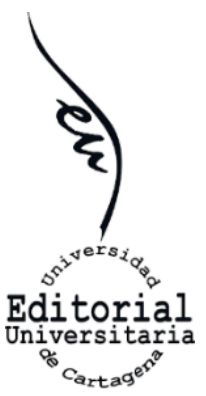

Stefany Cárdenas Martínez

Universidad de la Amazonia-Colombia, st.cardenas@udla.edu.co

Jhonier David Anacona Ortiz ${ }^{2}$ iD

Universidad de la Amazonia-Colombia, jh.anacona@udla.edu.co

Edwin Eduardo Millán Rojas 3 iD

Universidad de la Amazonia-Colombia, e.millan@udla.edu.co

\section{RESUMEN}

El punto de inicio de esta revisión de literatura está establecido bajo las categorías de colectivos de víctimas e inserción laboral enfocada a las problemáticas del conflicto armado en Colombia. Para la búsqueda de referencias se utilizó las bases de datos Scielo, Web of Science, Scopus, entre otras, debido que las premisas de colectivos de víctimas e inserción laboral están enfocadas al objetivo principal el cual se basa en describir los colectivos de las víctimas en aspectos sociodemográficos, formación, trayectorias laborales y expectativas de inserción laboral; fue necesario ampliar las opciones de búsqueda hacia medios nacionales enfatizando en medios locales como periódicos, revistas, informes nacionales y páginas web del Estado colombiano, los cuales tenían temáticas relevantes para la recolección información, así obteniendo un consolidado de 61 referencias para la elaboración de este documento.

Palabras clave: Guerra; problemas sociales; paz; psicología social; mujer en la política.

\footnotetext{
${ }^{1}$ Estudiante de Ingeniería de Sistemas de la Universidad de la Amazonia en la ciudad de Florencia en el departamento del Caquetá. Integrante del Grupo de Investigación de Gestión del Conocimiento, Informática, Electrónica y Comunicaciones GIECOM de la Universidad de la Amazonia

${ }^{2}$ Estudiante de Ingeniería de Sistemas de la Universidad de la Amazonia en la ciudad de Florencia en el departamento del Caquetá. Integrante del Grupo de Investigación en Informática, Innovación y Tecnología de la Universidad de la Amazonia -GITUA-.

${ }^{3}$ Investigador asociado según Colciencias, (2017), Doctor en Ingeniería, titulo otorgado por la Universidad Distrital Francisco José de Caldas (2016), Magíster en Ciencias de la información y las Comunicaciones de la Universidad Distrital Francisco José de Caldas (2012), Especialista en Ingeniería de Software de la Universidad INCCA de Colombia (2006) e Ingeniero de Sistemas de la Universidad Distrital Francisco José de Caldas, (2004), es docente asociado de la Facultad de Ingeniería de la Universidad de la Amazonia, Director del Grupo de Investigación en Informática, Innovación y Tecnología de la Universidad de la Amazonia -GITUA-
} 


\begin{abstract}
The starting point of this literature review is established under the categories of groups of victims and labor insertion focused on the problems of the armed conflict in Colombia. For the search of references, the databases Scielo, Web of Science, Scopus, among others were used, because the premises of victims and labor insertion groups are focused on the main objective which is based on describing the groups of victims in sociodemographic aspects, training, career paths and job placement expectations, it was necessary to expand the search options to national media emphasizing local media such as newspapers, magazines, national reports and websites of the Colombian state, which had relevant topics for the collection of information, thus obtaining a consolidated 61 references for the preparation of this document.
\end{abstract}

Keywords: War; social problems; peace; social psychology; woman in politics.

\title{
INTRODUCCIÓN
}

Desde hace 50 años Colombia, país latinoamericano ha sido víctima del conflicto armado interno; actualmente realiza una transición entre este problema, categorizado como uno de los más longevos de la región, y la paz, la cual es el objetivo por alcanzar con el acuerdo de paz establecido por la Oficina del Alto Comisionado para la Paz Colombia (2016) (Niño González, 2017).

Una de las principales resultados de la guerra son las víctimas que va dejando a su paso, las cuales han tenido que formar gupos de apoyo para reclamar sus derechos y apaciguar las cicatrices de guerra dejadas no solo de forma física, sino también psicológica; estos son llamados: colectivos de víctimas (Moreno Posada, 2015). Además, el desempleo de quienes en algún momento hicieron parte de las filas de los grupos armados al margen de la ley, por ello es de alta prioridad la creación de estrategias en pro de la inserción laboral de estas personas en el mercado colombiano (Gómez López \& Lesmes Romero, 2017).

En este artículo encontrará la investigación bibliográfica realizada respecto a las dos categorías mencionadas anteriormente (colectivos de víctimas e inserción laboral), para dar cumplimiento al objetivo: describir los colectivos de las víctimas en aspectos sociodemográficos, formación, trayectorias laborales y expectativas de inserción laboral, siendo este objetivo asignado desde el proyecto de Minciencias aprobado en la convocatoria 808 del 2018, el cual lleva como título Estrategias socioculturales y ambientales para el fomento del eco-turismo comunitario en el municipio de Florencia Caquetá, creado en la Universidad de la Amazonia. (“Universidad hacia el postconflicto”).

\section{METODOLOGíA}

El presente artículo es una revisión bibliográfica exploratoria-descriptiva con enfoque sistemático, la cual permitió a través de la investigación exploratoria tener una visión aproximada sobre los colectivos de víctimas a nivel nacional y departamental, así como la relación de estos con la inserción laboral en el país, 
para posteriormente enfocarse de forma descriptiva donde fue posible especificar las propiedades y características de cada uno de estos (Arias Odón, 2012; Hernandez Sampieri, 2018), además se optó por un enfoque sistemático para mapear y revisar no solo los hechos sobre el tema, sino también donde y de qué manera se han realizado (Manchado Garabito, y otros, 2009).

\section{Criterios de inclusión}

Los documentos seleccionados para la obtención de esta revisión bibliográfica fueron elegidos de acuerdo a criterios como: el año de publicación, el cual debía estar entre el rango de los años 2014 y 2019; el ámbito geográfico, preferiblemente nacional; $y$, por último, los temas tratados en cada documento, considerados con aportes significativos para así llegar a cumplir con el objetivo macro de la investigación.

\section{Fuentes de información y estrategias de búsqueda}

Las fuentes de información utilizadas fueron artículos y libros (en primer lugar) de bases de datos como: Scielo, Web of Science, Scopus, entre otras, las cuales son bases de datos indexadas y portadoras de fuentes de información confiable, pero debido al ámbito geográfico escogido, el hallazgo de documentos contextualizados era de baja cantidad, por lo tanto se tuvo que recurrir a medios locales, como periódicos, revistas, informes nacionales, páginas web reconocidas, los cuales brindaban una información enfocada en la problemática manejada específicamente en el pueblo colombiano.

\section{Ecuaciones de búsqueda}

Las categorías de búsquedas utilizadas para realizar la búsqueda en las fuentes de información anteriormente mencionadas, fueron: colectivos de víctimas, colectivos de víctimas en el Caquetá e inserción laboral en el postconflicto colombiano, las cuales permitían abarcar toda la temática base manejada en el artículo.

\section{Selección y clasificación}

Durante la búsqueda de los documentos se iba realizando un filtro a través de la revisión del título, resumen y/o conclusiones, los cuales permitían categorizarlo como pertinente o no pertinente dependiendo de su grado de ajuste a la temática de investigación.

Los documentos seleccionados fueron gestionados con ayuda de las herramientas: Mendeley, la cual permite el manejo de los metadatos para facilitar la tarea de referenciación de cada uno de estos, y de una plantilla hecha en Excel, en donde se organizó la información teniendo en cuenta el aporte significativo de la información en el tema tratado de acuerdo a las siguientes preguntas:

- ¿Qué se investiga?

- ¿Quién lo hace?

- ¿Dónde se produce la información?

- ¿En qué periodo de tiempo? 


\section{COLECTIVO DE VÍCTIMAS}

Colombia ha sido poseedor del conflicto armado durante aproximadamente 50 años a cargo de grupos militantes como: las Fuerzas Armadas Revolucionarias Colombianas (FARC), el ELN (Ejecito de Liberación Nacional), el EPL (Ejército Popular de Liberación) y el M-19 (Movimiento 19 de abril), los cuales surgieron en el siglo XX y se revelaron en forma armada contra el Estado (Jaramillo Bustamante, 2015), dando como resultado la formación de hechos victimizantes como: violencia corporal sexual, masacres indiscriminadas basadas en homicidios efectuados a la comunidad forzando a estos al desplazamiento forzado de sus campos a las ciudades (Hewitt Ramírez, y otros, 2016), alcanzando un impacto de violencia de manera directa a aproximadamente el 15\% de la población colombiana (Moreno Camacho \& Díaz Rico, 2017).

Los conflictos son ineludibles en la interacción social y nacen en todos los niveles reconociendo su grado e intensidad en cada situación, alcanza características diferenciadas (Calderón Rojas, 2016), no obstante, gracias a ordenanzas estatales como la Ley 1148 de 2011, la cual según García \& Muñoz (2011) establece parámetros de cuidado y ayuda humanitaria a las víctimas de este conflicto armado interno y a los conatos de paz con las FARC perpetrados por el país que se presentan desde el gobierno del presidente electo Andrés Pastrana en el año 1999, con una duración aproximada de tres años, el cual falló principalmente porque este no presentaba ningún avance, mientras la guerrilla de las FARC se fortalecía militarmente (Centro Nacional de Memoria Histórica, 2009); en el año 2012 en el periodo de mando del entonces presidente Juan Manuel Santos se ejecutó un nuevo un acuerdo de paz, mediante el cual se pretende la dejación de armas del mencionado grupo y se negocie una paz duradera (Jaramillo Bustamante, 2015), permitiendo el reconocimiento a la pluralidad de las víctimas del conflicto y la representación de la violencia sufrida en sus regiones y por los habitantes de las mismas (Guglielmucci, 2015).

En este contexto, de acuerdo con Guglielmucci (2017) los conceptos de víctima y victimarios, son una forma de reconocer el sufrimiento utilizándolo como una táctica para demandar a las entidades encargadas de sus casos y así de alguna manera obtener los recursos asignados para estos; entendiendo el concepto de derecho como una manera de reconocimiento recíproco, extendido a las personas libres, equitativamente cognitiva y responsables moralmente (Carrizo Sineiro \& Berger, 2019).

Desde el primero de enero de 1985 las víctimas del conflicto armado de origen colombiano fueron personas las cuales de forma colectiva individual han padecido algún perjuicio por problemáticas a partir de este tiempo, presentando a la víctima como inocente y cuyo derecho es un servicio (Moreno Posada, 2015).

De acuerdo con Martínez Lazcano, Cubides Cárdenas, \& Díaz Castillo (2016) exiten diferentes tipos de victimas: 
- Víctimas directas: son quienes hayan sufrido un daño económico, fisico, mental, emocional o de quienes hayan estado en peligro de sus derechos fundamentales como resultado del quebrantamiento a sus derechos.

- Victimas indirectas: son el nucleo familiar o las personas encargadas del cuidado de la víctima quienes compartan con esta.

- Victimas potenciales: son las personas que arriesgan su vida para socorrer a otra persona víctima.

Galindo Henao \& Parra Valencia (2015), Villa Gómez \& Insuasty Rodríguez (2015) y Villa, Londoño Díaz, \& Barrera Machado (2015), plantean al acompañamiento entre las personas pertenecientes a un grupo, en este caso: víctimas del conflicto armado, mediador para dar lugar a la noción de colectivos de víctimas, quienes a través de la capacidad de compartir, hablar, expresar su vivencia, los miedos, los sueños y las percepciones de la realidad a través de un proceso reflexivo, no solo desde la perspectiva del problema sino también desde las experiencias positivas posiblemente inmersas, forman redes a nivel social utilizadas como fuente de apoyo.

Aunque el gobierno ha realizado toda clase de ejercicios promotores de la aceptación y participación de los colectivos de víctimas, en los campos socioeconómicos y laborales, pretenden restaurar los bienes para todos esos campesinos quienes dejaron sus cultivos siendo este su principal entrada económica (Romero Acosta \& Banques Contreras, 2015), pero no ha sido posible dar cumplimiento a la premisa anterior y tampoco al proceso de resarcimiento integral de todas las personas damnificadas del conflicto armado colombiano (Molano Giraldo \& Rengifo Moreno, 2018), a causa de que estos grupos de víctimas han recurrido a manifestación trascendentales como hacer plantones en edificios públicos, al no ser escuchados utilizan un derecho fundamental como es la acción de tutela, la cual va dirigida a las personas empleados encargados de los proceso de reparación, así ejercer presión para una pronta respuesta (Rettberg, 2015).

Es de suma importancia acciones de indulgencia como el perdón, el cual permite la trascendencia y el aventajamiento sobre las actitudes sociales y los cambios en el comportamiento de aquellos quienes han sido victimarios. (Cortés, LópezLópez, Pérez D., \& Pineda-Marín, 2016), mientras tanto el castigo concebido en el contexto de un conflicto armado interno sin vencedores ni vencidos, siendo este un impedimento para la búsqueda de la paz (Ignacio Gómez, 2017) (Achutegui Otaolaurruchi, 2017), inhibiendo a personas con habilidades potentes en los diferentes campos gubernamentales, debido al auge sobre la participación política de los miembros de grupos al margen de la ley en cuestiones de liderazgo comunitario como consecuencia a la elevada exposición de violencia de la cual han sido partícipes (Batty \& M'Cormack-Hale, 2019).

Las mujeres han sido una de las principales víctimas de la violencia social, postulando como principal causa la "vulnerabilidad" presentado por este género (Gracia Ibáñez, 2015), sin embargo, de acuerdo con Mercedes Andrade (2015) en el ámbito del conflicto armado interno, fueron diversas las formas de ataque 
integral vivido por ellas, pero el principal fue la viudez, el cual provocó en un país tercermundista el descenso del sustento económico de estas, y un fácil acceso a la violencia carnal para quienes juegan el papel de victimarios, no obstante gracias a la popularidad de las mujeres en este ámbito les fue posible cumplir un importante papel político, un ejemplo de esto es: el movimiento o colectivo denominado Ruta Pacífica, creado en 1996 bajo la premisa de repudio al maltrato y violación masiva ocasionada en los municipios de Urabá, teniendo como principios fundamentales la búsqueda de la justicia y no repetición en estas comunidades (Sánchez Blake, 2016).

De igual forma, han sido creados otros colectivos similares al anterior, con fines restituyentes y de apoyo al posconflicto armado colombiano:

- Madres de la Candelaria, es una organización formada en el año 1999, como la unión de familiares víctimas de desaparición forzada, secuestro, desplazamiento, homicidio, masacres, entre otros: con el fin de buscar la verdad, la reparación, la justicia y la garantía de no repetición (Londoño \& Franco, 2018).

- Mujeres Caminando por la Verdad, tiene su origen en el año 2002 a causa de la Operación Orión desarrollada en la Comuna 13 de Medellín donde un gran número de personas fueron desaparecidas; se consolida en el año 2006 por medio de movilizaciones contra el entonces presidente Álvaro Uribe, con el fin de realizar la búsqueda de personas víctimas de desaparición forzada durante la operación ya mencionada (Juárez Rodríguez, Restrepo Echavarría, \& Botero Escobar, 2017).

- Las Madres de Soacha, son un grupo de madres quienes reclaman por la muerte de sus hijos, ocasionada por el ejército de Colombia en el año 2008, quienes los hicieron pasar por falsos positivos (Quintar, 2015).

- Mujeres de la masacre del Naya, son un grupo de mujeres indígenas viudas a causa de hechos violentos ocurridos a partir del año 2001, como: desplazamiento forzado, masacres, y despojo de tierras, a manos de las FARC (Díaz Bonilla, 2016).

El departamento del Caquetá no ha estado exento de la violencia, al contrario ha sido uno de los lugares donde el conflicto armado nacional ha estado más arraigado, por factores como: el espesor de sus selvas, lugares facilitadores de la clandestinidad necesaria para llevar a cabo masacres y actos de violencia de gran magnitud sin mayor presencia de testigos (Vásquez, 2014); hechos que a su paso han marcado de forma negativa a gran parte de la población local y por ello víctimas, quienes aún siguen con vida, han sido promulgadoras de la creación de colectivos de víctimas.

En la

Tabla 1 se describirán los colectivos más relevantes clasificados por su lugar de origen y donde además se mencionan las causas de origen de cada uno de estos: 
Tabla 1 .Colectivos de víctimas en el Caquetá

\begin{tabular}{|c|c|c|}
\hline Lugar & Colectivo & Causas \\
\hline \multirow{2}{*}{$\begin{array}{l}\text { Belén de los } \\
\text { Andaquíes }\end{array}$} & $\begin{array}{l}\text { Comunidad Puerto } \\
\text { Torres }\end{array}$ & $\begin{array}{l}\text { Confinamiento, amedrentamiento, tortura, } \\
\text { asesinato y desaparición forzada de personas } \\
\text { (Pinilla S, 2019) }\end{array}$ \\
\hline & $\begin{array}{l}\text { Comunidad El } \\
\text { Portal La Mono }\end{array}$ & $\begin{array}{l}\text { Desplazamiento de la población por conflicto } \\
\text { armado en los años } 2000 \text { a } 2004 \text { (Unidad para la } \\
\text { Atención y la Reparación Integral a las Víctimas, } \\
2018 \text { ) }\end{array}$ \\
\hline $\begin{array}{l}\text { Cartagena } \\
\text { del Chairá }\end{array}$ & $\begin{array}{l}\text { Comunidad de la } \\
\text { vereda Peñas } \\
\text { Coloradas }\end{array}$ & $\begin{array}{l}\text { Desplazamiento masivo y amedrentamiento de la } \\
\text { población en el año } 2004 \text { (Bustamante Gutiérrez, } \\
\text { 2016) }\end{array}$ \\
\hline \multirow{10}{*}{ Florencia } & $\begin{array}{l}\text { Resguardo } \\
\text { Honduras }\end{array}$ & $\begin{array}{l}\text { Desaparición, desplazamiento, masacres (Cadavid } \\
\text { Plitt, 2016) }\end{array}$ \\
\hline & $\begin{array}{l}\text { Cabildo Nasa } \\
\text { We'sx La Gaitana }\end{array}$ & $\begin{array}{l}\text { Masacres y desplazamiento forzado, en el año } \\
2001 \text { (Asociación de cabildos Indígenas del Norte } \\
\text { del Cauca, 2018) }\end{array}$ \\
\hline & $\begin{array}{l}\text { Cabildo Indígena } \\
\text { Muidomeni } \\
\text { (Naceda Inena) } \\
\end{array}$ & $\begin{array}{l}\text { Desplazamiento y amedrentamiento a la } \\
\text { comunidad (Defensoría del Pueblo, Colombia, } \\
\text { 2016) }\end{array}$ \\
\hline & $\begin{array}{l}\text { Resguardo Indígena } \\
\text { Páez, El Líbano }\end{array}$ & $\begin{array}{l}\text { Desplazamiento forzado (Equipo nacional plan de } \\
\text { salvaguarda y equipo de apoyo técnico y } \\
\text { profesional, 2014) }\end{array}$ \\
\hline & $\begin{array}{l}\text { Comunidad del } \\
\text { Cabildo Indígena } \\
\text { Monaya Buinaima }\end{array}$ & $\begin{array}{l}\text { Este cabildo liderado por Luz Meyi Patiño } \\
\text { actualmete, fue víctima de desplazamiento } \\
\text { forzado, según Garcés Amaya (2018) }\end{array}$ \\
\hline & $\begin{array}{l}\text { Resguardo San } \\
\text { Pablo del Para }\end{array}$ & $\begin{array}{l}\text { Desplazamiento forzado en la década del ochenta } \\
\text { (Unidad para la Atención y Reparación Integral a } \\
\text { las Víctimas, 2018; Ministerio del Interior, 2012) }\end{array}$ \\
\hline & $\begin{array}{l}\text { Cabildo Indígena } \\
\text { Embera Drua }\end{array}$ & $\begin{array}{l}\text { Desplazamiento forzado sobre los años 2010, } \\
2011 \text { y parte del } 2012 \text { (Mendes Cruz, en 2012; } \\
\text { Cabrera Marroquín \& Dominguez García, 2018) }\end{array}$ \\
\hline & $\begin{array}{l}\text { Resguardo San } \\
\text { Pablo del Para }\end{array}$ & $\begin{array}{l}\text { Desplazamiento forzado en la década del ochenta } \\
\text { (Unidad para la Atención y Reparación Integral a } \\
\text { las Víctimas, 2018; Ministerio del Interior, 2012) }\end{array}$ \\
\hline & $\begin{array}{l}\text { Cabildo Indígena } \\
\text { Urbano Uitoto } \\
\text { Jurama }\end{array}$ & $\begin{array}{l}\text { Desplazamiento y amedrentamiento a la } \\
\text { población (Defensoría del Pueblo, Colombia, } \\
\text { 2016) }\end{array}$ \\
\hline & $\begin{array}{l}\text { Cabildo Indígena } \\
\text { Coreguaje Chaibaju }\end{array}$ & $\begin{array}{l}\text { Reclutamiento, desplazamiento y falsos positivos } \\
\text { (Resguardo Indígena Agua Negra, 2014) }\end{array}$ \\
\hline \multirow[t]{2}{*}{$\begin{array}{l}\text { La } \\
\text { Montañita }\end{array}$} & $\begin{array}{l}\text { Comunidad de la } \\
\text { Unión Peneya }\end{array}$ & $\begin{array}{l}\text { En el año } 2004 \text { los habitantes de esta comunidad } \\
\text { tuvieron que desplazarse forzadamente de sus } \\
\text { tierras (Alcaldía de La Montañita, 2012; El } \\
\text { Espectador, 2018) }\end{array}$ \\
\hline & $\begin{array}{l}\text { Cabildo Indígena } \\
\text { Lulumoy }\end{array}$ & $\begin{array}{l}\text { Desplazamiento forzado (Ministerio del Interior, } \\
\text { 2015) }\end{array}$ \\
\hline \multirow{2}{*}{ Milán } & $\begin{array}{l}\text { Resguardo Indígena } \\
\text { Agua Negra del } \\
\text { municipio de Milán }\end{array}$ & $\begin{array}{l}\text { Desplazamiento forzado (Unidad para la Atención } \\
\text { y Reparación Integral a las Víctimas, 2018) }\end{array}$ \\
\hline & $\begin{array}{l}\text { Resguardo Indígena } \\
\text { Coreguaje de San } \\
\text { Luis }\end{array}$ & Desplazamiento forzado (Prada Céspedes, 2018) \\
\hline
\end{tabular}


Amparo Micolta León, Maritza Charry Higueras, Maria Cenide Escobar Serrano

\begin{tabular}{|c|c|c|}
\hline $\begin{array}{l}\text { San José del } \\
\text { Fragua }\end{array}$ & $\begin{array}{l}\text { Cabildo Indígena } \\
\text { Jaieni Diona Portal } \\
\text { la Fraguita }\end{array}$ & $\begin{array}{l}\text { Desplazamiento forzado (Unidad para la Atención } \\
\text { y la Reparación Integral a las Víctimas, 2018) }\end{array}$ \\
\hline \multirow{3}{*}{$\begin{array}{l}\text { San Vicente } \\
\text { del Caguán }\end{array}$} & $\begin{array}{l}\text { Cabildo Emberá } \\
\text { Chamí La Pradera }\end{array}$ & $\begin{array}{l}\text { Desplazamiento forzado (Acuña Romero, León } \\
\text { Valencia, \& Angarita Isaza, 2018) }\end{array}$ \\
\hline & $\begin{array}{l}\text { Resguardo Indígena } \\
\text { Llanos del Yarí- } \\
\text { Yaguará }\end{array}$ & $\begin{array}{l}\text { Desplazamiento forzado y desaparición de líderes } \\
\text { (Ministerio del Interior, 2012) }\end{array}$ \\
\hline & $\begin{array}{l}\text { Resguardo Indígena } \\
\text { Embera-Chamí La } \\
\text { Libertad II }\end{array}$ & Desplazamiento forzado (Pulido Marciales, 2015) \\
\hline Solano & $\begin{array}{l}\text { Cabildo Indígena } \\
\text { Ismuina }\end{array}$ & $\begin{array}{l}\text { Amenaza, desplazamiento forzado, homicidio, } \\
\text { discriminación y masacres (Defensoría del } \\
\text { Pueblo, Colombia, 2016) }\end{array}$ \\
\hline Valparaíso & $\begin{array}{l}\text { Comunidad de } \\
\text { Santiago de la } \\
\text { Selva }\end{array}$ & $\begin{array}{l}\text { Desplazamiento forzado, masacres y } \\
\text { reclutamiento (Ramirez Aldana, 2019) }\end{array}$ \\
\hline
\end{tabular}

Fuente: Elaboración propia

Se hace visible en la tabla anterior y en el Gráfico 1 cómo la capital del departamento ha sido una de las más afectadas por el conflicto armado interno, además de proveer herramientas de reparación como lo son los colectivos y resguardos creados para aquellos quienes fueron víctimas de estos hechos y sanen sus cicatrices de guerra.

Gráfico 1 Número de colectivos de víctimas clasificados por municipios en el Caquetá

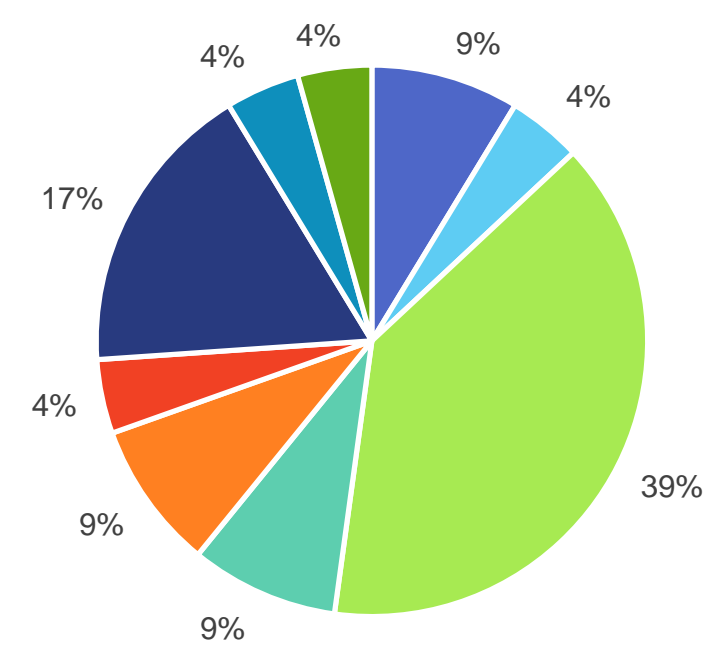

$$
\begin{aligned}
& \text { - Belén de los Andaquíes - Cartagena del Chaira = Florencia } \\
& \text { - La Montañita - Milán - San José del Fragua } \\
& \text { - San Vicente del Caguán - Solano - Valparaíso }
\end{aligned}
$$

Fuente: Elaboración propia

Además es notorio que la principal razón del conflicto armado nacional es el desplazamiento obligatorio (ver Gráfico 2) factor por el cual Colombia es llamada una nación desplazada, ocupando el segundo puesto a nivel mundial con 
una alta tasa de desplazado por problemáticas internas (Centro Nacional de Memoria Histórica, 2015).

Gráfico 2. Causas de creación de colectivos

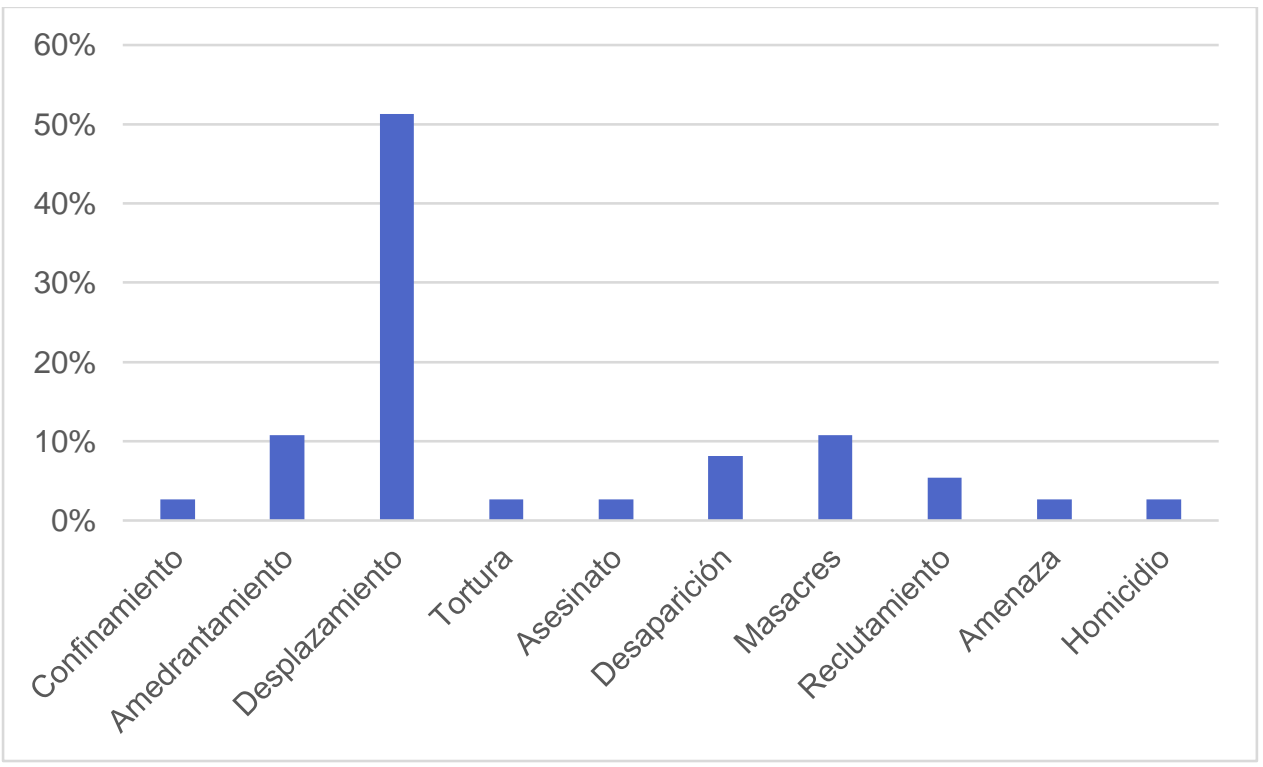

Fuente: Elaboración propia.

\section{INSERCIÓN LABORAL}

El correcto desarrollo del postconflicto colombiano demanda la desintegración de los grupos armados sobre factores ilegales, para ello es indispensable, según Mesa (2017): la desmovilización, la reintegración, dejación de armas y la reinserción a la vida civil. Los objetivos de la aplicación de estos factores, son tres según Pinto Borrego, et al., (2003) citado en Crespi Vallbona, Galeas Ortiz, \& López Zúñiga (2019, págs. 158-159):

- Los miembros de los grupos desmovilizados tendrán participación política.

- Las personas desmovilizadas contarán con seguridad, opciones de nueva convivencia social en los entornos de conflicto.

- Para la vida productiva contará con el desarrollo económico y su recuperación.

Este último será el enfoque de estudio de esta sección, el cual será orientado al sector laboral.

Tratar de incluir al sistema laboral a aquellas personas pertenecientes a grupos insurgentes y los cuales se acogieron de buena obra a las autoridades pertinentes para definir su estado y así lograr reincorporarse a la vida sin armas (Molinares Hassan, 2016) desata un gran problema dada la reacción tomada por la sociedad frente al pasado de estas personas (Gómez López \& Lesmes Romero, 2017); comúnmente la estigmatización social es el principal factor en contra de este proceso (Restrepo Pineda, Giraldo Arboleda, Buenaventura Ibargüen, \& 
Ameriles Calderón, 2017), sin embargo existen otros factores como el nivel bajo de escolaridad (ver Gráfico 3) de los desmovilizados, lo cual los deja en desventaja frente a las demás personas, debido a la vinculación temprana de estos en grupos armados ilegales, arrebatándole no solo su niñez, sino también su derecho a la educación (Bácares Jara, 2015).

Gráfico 3. Población desmovilizada por nivel de escolaridad 2002-2016

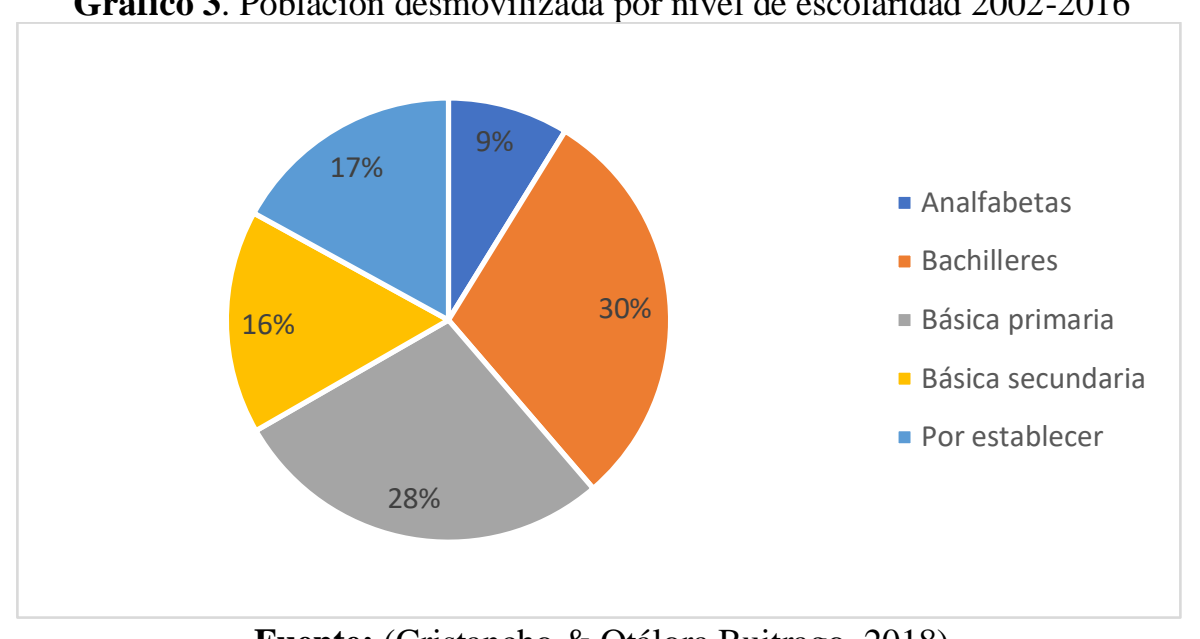

Fuente: (Cristancho \& Otálora Buitrago, 2018)

Garay Gutiérrez (2016) expone a través del Gráfico 4 cómo el número de cursos técnicos realizados por los individuos después de su respectiva desmovilización es poca y de tal forma se ve afectado el sector académico, el cual tiene un papel trascendental en el marco laboral, ya que a través de este se capacitan las personas para ayudar al mejoramiento social presentado como una necesidad en los diferentes estados alrededor del mundo, a través de la inserción laboral (Dapía Conde \& Fernández González, 2018), la cual de acuerdo con Pinto Baleisan (2016) es definida como el resultado del desarrollo de estrategias y acciones concretas, realizadas por individuos para encontrar y mantener un trabajo remunerado de acuerdo a la formación obtenida. Cabe destacar también, las iniciativas creadas por las instituciones educativas quienes van en contra de la exclusión y discriminación laboral, para promover la inserción al mercado de los estudiantes recién egresados y quienes tienen poca o nula experiencia laboral (Zwerg Villegas \& Jiménez Aguilar, 2015).

Gráfico 4. Formación para el trabajo en desmovilizados 


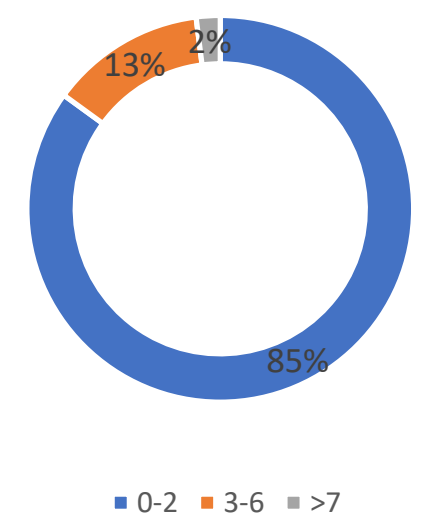

Fuente: (Garay Gutiérrez, 2016)

Las pequeñas y medianas empresas (PyMEs) acarrean la responsabilidad de servir como soporte para la inclusión laboral de la población en proceso de reinserción, sin embargo muchas de ellas no generan estrategias para reclutar a esta población (Maya Cabrera, Flórez Rodríguez, \& Matabanchoy Tulcán, 2019) y/o desconocen las políticas de Responsabilidad Social Empresarial (RSE), las cuales según Jiménez Sierra \& Pérez Suescún (2016): deben garantizar el cuidado de las personas civiles por encima de los deberes del Estado, donde se priorice las personas causantes del conflicto (Barrios Hernández, 2016); a pesar del desconocimiento de estas, algunas empresas las aplican de forma indirecta, capacitando a sus empleados, contribuyendo con dos causas como son los problemas sociales y el medio ambiente, por ejemplo la inserción laboral de la persona es acogidas en el marco del posconflicto, de acuerdo con Sepúlveda Romero, Moreno Martínez, Tovar Mesa, Franco Villada, \& Villarraga Tole (2015).

De acuerdo al estudio investigativo realizado por Castillo Castillo \& Roncancio García (2018) las víctimas del conflicto armado presentan inclinación por ciertas vocaciones (ver Tabla 2), en donde se evidenció una mayor preferencia por los trabajos mecánicos mediante los cuales se fortalecen habilidades como: innovación, creatividad, planeación y diseño, asi se facilita el ejercer cargos laborales dentro de las empresas. 
Tabla 2. Vocaciones de las víctimas del conflicto armado colombiano

\begin{tabular}{|l|l|l|}
\hline Vocación & Descripción & Porcentaje \\
\hline Cálculo & $\begin{array}{l}\text { Para las personas que les gusta trabajar con } \\
\text { razonamientos numéricos. }\end{array}$ & $10 \%$ \\
\hline $\begin{array}{l}\text { Científico- } \\
\text { fisico }\end{array}$ & $\begin{array}{l}\text { Para las que se interesan por la } \\
\text { investigación de la propiedad de la materia, } \\
\text { los cuerpos y la elaboración de leyes } \\
\text { naturales. }\end{array}$ & $10 \%$ \\
\hline $\begin{array}{l}\text { Científico- } \\
\text { biológico }\end{array}$ & $\begin{array}{l}\text { Personas con inclinación a la investigación } \\
\text { de la vida humana. }\end{array}$ & $10 \%$ \\
\hline Mecánico & $\begin{array}{l}\text { Indica interés para trabajar con máquinas y } \\
\text { herramientas de tipo industrial, objetos } \\
\text { mecánicos, eléctricos, muebles, equipo de } \\
\text { cirugia, etc. }\end{array}$ & $28 \%$ \\
\hline $\begin{array}{l}\text { Servicio } \\
\text { Social }\end{array}$ & $\begin{array}{l}\text { Indica alto grado de interés por servir a los } \\
\text { demás. }\end{array}$ & $4 \%$ \\
\hline Literario & $\begin{array}{l}\text { Indica placer en la lectura y en la expresión } \\
\text { de ideas propias en forma oral o escrita. }\end{array}$ & $11 \%$ \\
\hline Persuasivo & $\begin{array}{l}\text { Indica el agrado por imponer su punto de } \\
\text { vista, convencer y manejar a los demás. }\end{array}$ & $12 \%$ \\
\hline Artístico & $\begin{array}{l}\text { Indica el gusto por las creaciones de tipo } \\
\text { manual. }\end{array}$ & $8 \%$ \\
\hline Musical & $\begin{array}{l}\text { Denota un marcado gusto por cantar, tocar } \\
\text { instrumentos, etc. }\end{array}$ & $7 \%$ \\
\hline
\end{tabular}

Fuente: (Castillo Castillo \& Roncancio García, 2018)

A pesar del innegable rechazo social hacia los desmovilizados de las FARC, estudios como los de Rubio Rodríguez, Rodríguez Barrero, \& Moreno Espinosa (2018) demuestran que el 50\% de la población encuestada se presenta positiva frente a la equidad de goce en oportunidadesy derechos de los desmovilizados al igual que las demás personas, aún así el gobierno debe seguir trabajando en terminos de una convivencia pacífica y solidaria para la población que aún no perdona (ver Gráfico 5).

Gráfico 5. Igualdad de derechos y oportunidades. 


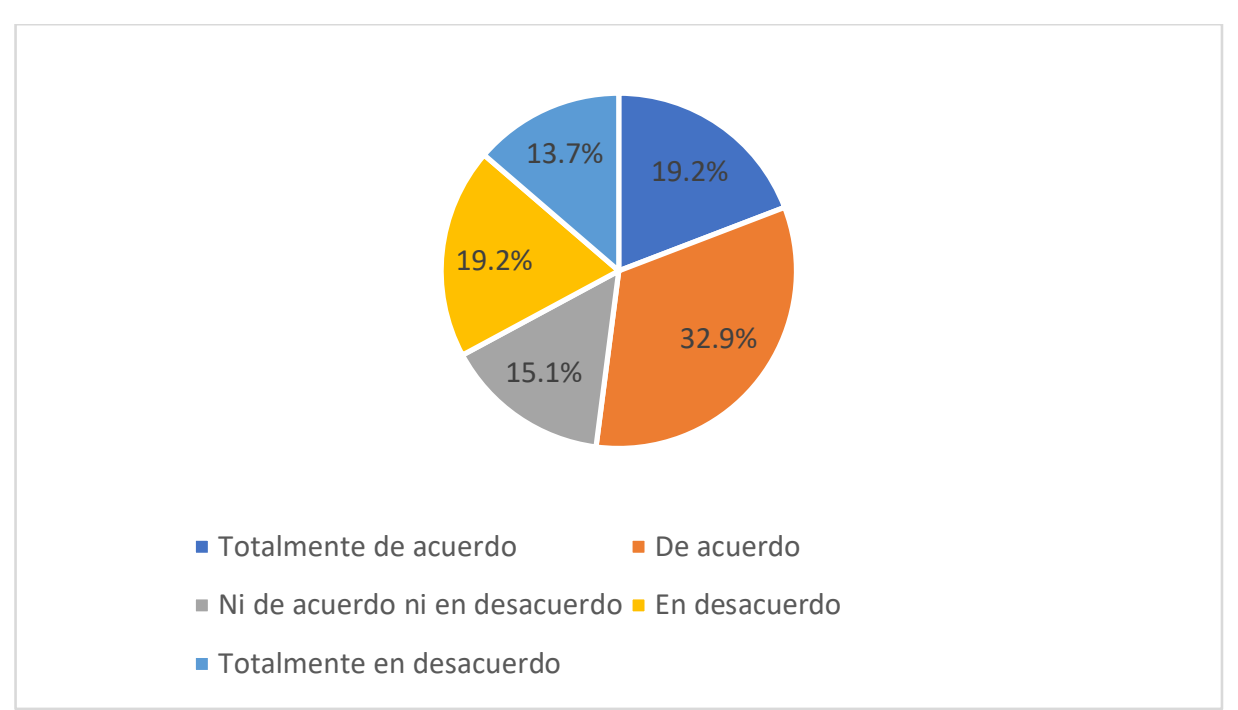

Fuente: (Rubio Rodriguez, Rodriguez Barrero, \& Moreno Espinosa, 2018)

\section{CONCLUSIONES}

Durante la historia de Colombia, esta ha sido azotada por el conflicto armado; su liberación de la colonización española se dio gracias a la pelea dada por los llamados en aquel tiempo: "Criollos", los cuales eran conformados por las personas del campo quienes pelearon por sus derechos. Desde ese entonces la fuerza de pelea se ha utilizado para la obtención del poder y rechazo contra los entes del gobierno. Hace aproximadamente 50 años el conflicto armado interno colombiano creció teniendo repercusiones en toda la nación; las olas delincuenciales azotaron fuertemente la integridad social y política, ocasionando problemáticas a todas las poblaciones.

Desde la firma del acuerdo de paz firmado en el Teatro Colon en 2016 se ha presentado una disminución en los ataques a las personas civiles y a las fuerzas armadas colombianas, esto ha sido una evolución trascendental en la historia de Colombia. Gracias a este acuerdo con las FARC sus integrantes se acogieron a lo pactado, brindando tranquilidad a las comunidades víctimas de estos ya que al realizar su entrega brindaban información de muchos actos delincuenciales especialmente la información necesaria para saber el paradero de personas desparecidas, información que era necesaria para las familias teniendo como propósito darle santa sepultura y así poder descansar de la incertidumbre causada. Teniendo como fundamento el objetivo principal el cual se basa en describir los colectivos de las víctimas en aspectos sociodemográficos, formación, trayectorias laborales y expectativas de inserción laboral, se logra determinar que la creacion de los colectivos de víctimas tienen su origen comun en el desplazamiento forzado, donde este fue una carácterística principal en los grupos armados ilegales que obligaban a las personas a salir de sus propiedades, abandonando sus tierras, cultivos y bienes, ocasionando que muchos tuvieran que salir de estas zonas, ya que fueron muchas las personas que sufrieron por esta causa se agruaban en zonas asi formando los colectivos de víctimas, conviertiendo un daño comun en colectivo, organizándose con el fin de pelear por sus derechos. 
Tras el avance logrado en el acuerdo de paz muchas personas las que dejaron las armas se han encontrado con un problema común y es la aceptación por la sociedad, la cual no acepta a los reinsertados en la vida cotidiana de ellos, ya que estos hicieron muchos daños a las sociedades, es por esto que presentan repudio e indignación por una sociedad llena de temor. Este repudio no solo es el único problema, a esto se le debe sumar la problemática de los excombatientes por entrar a la vida laboral, las expectativas por ingresar en estos campos son casi nulos ya que muchas empresas en sus políticas se reservan el derecho de admisión para incorporar a este tipo de persona, además la falta de educación de estos desmovilizados hace que no puedan optar por cargos importantes, ya que no cuentan con conocimientos necesarios y solo puedan aspirar a trabajos poco remunerados. Este problema de educación puede tener consecuencias negativas enfocadas a la dejación de armas, donde estas personas al no tener una buena entrada económica y calidad de vida pueden volver a reincidir en sus antiguas acciones delincuenciales.

Como trabajo futuro se podría especificar la relación existente entre los estragos de la guerra y las afecciones psicológicas ocasionadas por la guerra hacia las víctimas de esta.

\section{REFERENCIAS BIBLIOGRÁFICAS}

Achutegui Otaolaurruchi, P. (2017). Victimización de los delitos de odio. Aproximación a sus consecuencias y a las respuestas institucional y social. Revista de victimología(5), 33-62. doi:10.12827/RVJV.5.02

Acuña Romero, E., León Valencia, K. T., \& Angarita Isaza, C. I. (2018). Identificación de la garantía o vulneración del Derecho al territorio de la comunidad indígena Embera Chamí Dachi Drua en situación de desplazamiento forzado, asentada en el corregimiento Campo Capote, municipio de Puerto Parra en Santander. Tesis de grado, Universidad Cooperativa de Colombia, Facultad de Derecho, Barrancabermeja. Recuperado el 2019, de http://repository.ucc.edu.co/bitstream/ucc/6636/1/2018_derecho_territorio_indig ena.pdf

Alcaldía de La Montañita. (2012). Plan integral para la población desplazada (PIU). Florencia. Recuperado el 2019, de http://cdim.esap.edu.co/bancomedios/documentos\%20pdf/lamonta\%C3\%B1itac aquetapiu2012..pdf

Arias Odón, F. G. (2012). El proyecto de investigación. Introducción a la metodología científica (Sexta ed.). (F. G. Arias Odón, Ed.) Caracas, Venezuela: Episteme. Recuperado el 2019, de https://books.google.com.co/books?id=W5n0BgAAQBAJ\&hl=es\&source=gbs_ navlinks_s

Asociación de Cabildos Indígenas del Norte del Cauca. (18 de 08 de 2018). El Naya: caminando la vida y la memoria. nasaacin.org. Recuperado el 2019, de https://nasaacin.org/el-naya-caminando-la-vida-y-la-memoria/ 
Bácares Jara, C. (2015). Los niños, niñas y jóvenes desvinculados de los grupos armados ilegales en Colombia: ¿Víctimas de la violencia política o sujetos del delito? $\quad$ Estudios $\quad$ socio-jurídicos, $\quad 7(2), \quad 233-262$. doi:dx.doi.org/10.12804/esj17.02.2015.07

Barrios Hernández, R. (2016). Responsabilidad social empresarial: el papel de las empresas en el postconflicto colombiano. Ensayos: Revista de Estudiantes de Administración de Empresas, 9(1), 45-56. Recuperado el 2019, de https://revistas.unal.edu.co/index.php/ensayos/article/view/61276

Batty, F., \& M'Cormack-Hale, F. (2019). "Do not Disturb the Peace!” Identities, Livelihoods and the Politics of Post-War Discontent in Sierra Leone. Journal of Asian and African Studies, 54(4), 533 -553. doi:10.1177/0021909618825355

Bustamante Gutiérrez, C. (23 de Noviembre de 2016). Sin retorno: memorias de un desplazamiento oculto en Caquetá. Radio Nacional de Colombia. Recuperado el 2019, de https://www.radionacional.co/especial-paz/sin-retorno-memoriasdesplazamiento-oculto

Cabrera Marroquín, A., \& Domínguez García, D. P. (2018). Diseño de un plan productivo integral para el Resguardo Dachi Drua, Comunidad Indígena Emberá Chamí, del municipio de Tuluá. Tesis de grado, Universidad del Valle, Tuluá. Recuperado el 2019, de http://hdl.handle.net/10893/12574

Cadavid Plitt, A. (2016). Límites y alcances de la ayuda humanitaria brindada por ACNUR en Colombia: caso del proyecto Construyendo soluciones sostenibles 2012-2015 en la comunidad indígena Emberá Chamí del Caquetá. 51. Bogotá D.C., Colombia: Universidad del Rosario. Recuperado el 2019, de http://repository.urosario.edu.co/handle/10336/13476

Carrizo Sineiro, C., \& Berger, M. (2019). Hacia una ley para el reconocimiento y garantía de derechos de los afectados ambientales. Prometeica - Revista de Filosofía y Ciencias, 59(18), 59-70. doi:10.24316/prometeica.v0i18.244

Castillo Castillo, D. A., \& Roncancio García, A. D. (2018). Descripción de perfiles ocupacionales y vocacionales para la reintegración a la vida laboral de víctimas del conflicto armado colombiano. Estudio de caso Unidad para las Víctimas. Revista Latinoamericana en Derechos Humanos, 29(1), 167-196. doi:https://doi.org/10.15359/rldh.29-1.7

Centro Nacional de Memoria Histórica. (2009). La masacre de El Salado: Esa guerra no era nuestra. Colombia: Grupo de Memoria Histórica. Obtenido de www.memoriahistorica-cnrr.org.co

Centro Nacional de Memoria Histórica. (2015). Una nación desplzada - Informe nacional del desplazamiento forzado en Colombia. Bogotá D.C., Colombia: Imprenta Nacional de Colombia. Recuperado el 2019, de http://www.centrodememoriahistorica.gov.co/descargas/informes2015/naciondesplazada/una-nacion-desplazada.pdf

Cortés, Á., López-López, W., Pérez D., C., \& Pineda-Marín, C. (2016). Comprensiones sobre el perdón y la reconciliación en el contexto del conflicto 
armado colombiano. Psychosocial Intervention, 252, 19-25. doi:10.1016/j.psi.2015.09.004

Crespi Vallbona, M., Galeas Ortiz, S. A., \& López Zuñiga, M. A. (2019). Desarrollo turístico inclusivo socialmente. El caso de los desmovilizados en la región del Chocó, Colombia. Cuadernos Geográficos, 58(1), 157-179. doi:http://dx.doi.org/10.30827/cuadgeo.v58i1.6759

Cristancho, L. A., \& Otálora Buitrago, A. (2018). Inclusión laboral de los desmovilizados del conflicto armado en Colombia. Tendencias Sociales(1), 169196. doi:https://doi.org/10.5944/ts.1.2018.21366

Dapía Conde, M. D., \& Fernández González, M. R. (2018). Educación social y escuela en España. A propósito de la formación e inserción laboral. Revista $\begin{array}{llll}\text { Iberoamericana de } & \text { 209-228. }\end{array}$ doi:https://doi.org/10.35362/rie7602857

Defensoría del Pueblo Colombia. (2016). Acciones de la Delegada de los Indígenas y Minorías Étnicas de la Defensoría del Pueblo Regional Caquetá 2015. Informe rendición de cuentas regional Caquetá, Florencia. Recuperado el 2019, de http://www.defensoria.gov.co/public/rendiciondecuentas/assets/regionalcaqueta2.pdf

Díaz Bonilla, P. A. (5 de Diciembre de 2016). Justicia en clave psicosocial: Miradas desde las voces de las mujeres viudas del Alto Naya. RevistaCambios y Permanencias(7), 85-112. Recuperado el 2019, de https://revistas.uis.edu.co/index.php/revistacyp/article/view/7018/7290

El Espectador. (25 de Octubre de 2018). El valiente retorno a su pueblo de la comunidad de la Unión Peneya (Caquetá). El Espectador. Recuperado el 2019, de https://www.elespectador.com/colombia2020/pedagogia/el-valiente-retorno-supueblo-de-la-comunidad-de-la-union-peneya-caqueta-articulo-857296

Equipo Nacional Plan de Salvaguarda y Equipo de Apoyo Técnico y Profesional. (2014). La nación Nasa en medio del conflicto armado. Derechos civiles y políticos y DIH. Plan de Salvaguarda de la Nación Nasa, 291. Colombia. Recuperado el 2019, de https://www.mininterior.gov.co/sites/default/files/p.s_nasa_version_preliminar.p df

Garay Gutiérrez, D. (2016). Probabilidad de reincidencia criminal en personas vinculadas al porceso de reintegración social en Bogotá. Trabajo de grado, Universidad Javeriana, Bogotá. Recuperado el 2019, de https://repository.javeriana.edu.co

Gómez López, C. A., \& Lesmes Romero, A. L. (Mayo de 2017). Reintegración laboral de los desmovilizados. "Diagnóstico a empresarios". Bogotá D. C.: Universidad Católica de Colombia. Recuperado el 2019, de https://repository.ucatolica.edu.co/bitstream/10983/14426/4/Reintegraci\%C3\%B 3n\%20Laboral\%20de\%20los\%20desmovilizados\%20\%20Diagnostico\%20a\%20 .pdf 
Gracia Ibáñez, J. (2015). Una mirada interseccional sobre la violencia de género contra las mujeres mayores. Oñati Socio-legal Series, 5(2), 547-569. Recuperado el 2019, de http://ssrn.com/abstract=2611006

Hernández Sampieri, R. (2018). Metodología de la investigación: las rutas cuantitativa, cualitativa y mixta (Primera ed.). México, México: McGraw-Hill Education. Recuperado el 2019, de https://books.google.com.co/books?id=5A2QDwAAQBAJ

Hewitt Ramírez, N., Juárez, F., Parada Baños, A. J., Guerrero Luzardo, J., Chávez, Y. M., Salgado Castilla, A. M., \& Vargas Amaya, M. V. (2016). Afectaciones psicológicas, estrategias de afrontamiento y niveles de resiliencia de adultos expuestos al conflicto armado en Colombia. Revista Colombiana de Psicologia, 25(1), 125-140. doi:10.15446/rcp.v25n1.49966

Jaramillo Bustamante, V. (2015). Conflicto armado en Colombia, el proceso de paz y la Corte Penal Internacional: Un estudio sobre la internacionalización del conflicto armado en Colombia y su búsqueda por encontrar la paz duradera. Journal of International Law, 6(2), 6-34. Obtenido de http://publicaciones.eafit.edu.co/index.php/ejil/article/view/3468

Jiménez Sierra, D., \& Pérez Suescún, L. (2016). La representación simbólica de la Responsabilidad Social Empresarial (RSE): El caso Santa Marta. Investigación e Innovación en Ingenierías, 4(2), 72-108. doi:https://doi.org/10.17081/invinno.4.2.2487

Juárez Rodríguez, J., Restrepo Echavarría, N. J., \& Botero Escobar, N. E. (2017). Los movimientos sociales de mujeres y su consolidación como interlocutor y actor político en la construcción de la paz en Medellín, Colombia. Izquierdas, (34), 124. doi:http://dx.doi.org/10.4067/S0718-50492017000300001

Londoño, J. C., \& Franco, R. E. (2018). Madres de La Candelaria: construyendo políticas públicas en contextos de violencia. Analecta política, 8(15), 315-338. Obtenido de https://dialnet.unirioja.es/servlet/articulo? codigo $=6673376$

Manchado Garabito, R., Tamames Gómez, S., López González, M., Mohedano Macías, L., D’Agostino, M., \& Veiga de Cabo, J. (Julio de 2009). Revisiones sistemáticas exploratorias. Medicina y seguridad del trabajo, 55(216), 12-19. Recuperado el 2019, de http://scielo.isciii.es/scielo.php?pid=S0465546X2009000300002\&script=sci_arttext\&tlng=pt

Maya Cabrera, D. C., Flórez Rodríguez, Y. N., \& Matabanchoy Tulcán, S. M. (2019). Retos del área de talento humano frente a la inserción laboral de personas en proceso de reintegración. Informes Psicológicos, 19(1), 163-180. doi:http://dx.doi.org/10.18566/infpsic.v19n1a09

Méndes Cruz, J. F. (2012). Entre excusas y desconocimietnos ¿El enfoque diferencial como la solución para las comunidades étnicas? Tesis doctoral, Pontificia Universidad Javeriana, Ciencia política y relaciones internacionales, Bogotá. Obtenido de http://hdl.handle.net/10554/7804 
Mihaela Spatarelu, E. (2015). Youth Insertion on Labor Market. Procedia Economics and Finance, 32(15), 1020-1026. doi:10.1016/S2212-5671(15)015634

Ministerio del Interior. (2012). Plan salvaguarda embera, asentamientos dispersos. Bogotá D.C. Recuperado el 2019, de https://www.mininterior.gov.co/sites/default/files/p.s_chami_y_katio_poblacion _dispersa_0.pdf

Ministerio del Interior. (2015). Diagnóstico participativo del estado de los derechos fundamentales del pueblo Pijao y líneas de acción para la contrucción de su plan de salvaguardia étnica. Recuperado el 2019, de MinInterior: https://www.mininterior.gov.co/sites/default/files/anexo_iv.pdf

Molano Giraldo, M., \& Rengifo Moreno, H. A. (2018). Participación política de víctimas ¿reivindicación o instrumentalidad? Nova et Vetera, 1(27), 48-75. doi: $10.22431 / 25005103.503$

Molinares Hassan, V. (2016). Protección a la libertad y seguridad personal en Colombia: avances de un proceso. Cuestiones constitucionales, 34, 65-102. doi:https://doi.org/10.1016/j.rmdc.2016.07.003

Moreno Posada, A. (2015). Las víctimas del conflicto armado colombiano en la Ley de Víctimas y Restitución de Tierras: apropiación y resignificación de una categoría jurídica. Perfiles Latinoamericanos, 23(46), 121-145. Recuperado el 2019, de http://www.scielo.org.mx/scielo.php?script=sci_arttext\&pid=S018876532015000200005

Niño González, C. A. (2017). Breve historia del conflicto armado en Colombia. $\begin{array}{lllll}\text { Revista de Paz y } & \text { Conflictos, } & \text { 10(1), }\end{array}$ doi:https://dialnet.unirioja.es/descarga/articulo/6103291.pdf

Pinilla S, A. C. (29 de Abril de 2019). Memorias de Puerto Torres, inspección de Belén de los Andaquíes en espera de la reparación colectiva. AmazoniaypaZ, pág. 3. Recuperado el 2019, de https://amazoniaypaz.com/puerto-torres-14-anosesperando-la-reparacion-colectiva/

Pinto Baleisan, C. (2016). Reflexiones sobre la inserción laboral de doctores en universidades chilenas. Fronteras, 3(1), 109-124. Recuperado el 2019, de http://publicacionescienciassociales.ufro.cl/index.php/fronteras/index

Prada Céspedes, E. (27 de Enero de 2018). Coreguaje: voces de un despojo. Obtenido de Agenda Propia: Un medio para la investigación independiente: https://www.agendapropia.co/content/coreguaje-voces-de-un-despojo

Pulido Marciales, M. (2015). Intervención institucional en la comunidad Embera Chamí: bajo la lupa crítica de la acción sin daño. Tesis de grado, Universidad Nacional de Colombia, Ciencias Humanas, Bogotá D.C. Recuperado el 2019, de http://www.bivipas.unal.edu.co/bitstream/123456789/721/1/TrabajoFinalMarisol Pulido.pdf 
Quintar, E. (2015). Memoria e historia desafíos a las prácticas políticas de olvido en América Latina. Revista El Ágora USB, 15(2), 375-391. Recuperado el 2019, de https://nbn-resolving.org/urn:nbn:de:0168-ssoar-458625

Ramírez Aldana, G. (22 de marzo de 2019). Santiago de la Selva, un pueblo transformado por el conflicto. Las 2 Orillas. Recuperado el 2019, de https://www.las2orillas.co/santiago-de-la-selva-un-pueblo-transformado-por-elconflicto/

Resguardo Indígena Agua Negra. (2014). Plan de salvaguarda - Pueblo Koreguaju. CRIOMC. Recuperado el 2019, de https://siic.mininterior.gov.co/sites/default/files/pueblo_koreguaje__diagnostico_comunitario.pdf

Restrepo Pineda, J. E., Giraldo Arboleda, M., Buenaventura Ibarguen, O. I., \& Ameriles Calderón, L. J. (Octubre de 2017). El proceso de inclusión laboral de los desmovilizados en la ciudad de Medellín y su área metropolitana. Logos Ciencia y Tecnología, 9(2), 10-26. Recuperado el 2019, de http://revistalogos.policia.edu.co/index.php/rlct/article/download/477/pdf

Rettberg, A. (2015). Ley de Víctimas en Colombia: Un balance. Revista de Estudios Sociales, 185-188. doi:10.7440/res54.2015.14

Romero Acosta, K. C., \& Banques Contreras, E. M. (2015). Revisión teórica sobre el post-conflicto: Una oportunidad para empoderar a mujeres víctimas de desplazamiento. Cultura, Educación y Sociedad, 6(1), 1-166. Recuperado el 2019, de

https://revistascientificas.cuc.edu.co/culturaeducacionysociedad/article/view/757

Rubio Rodríguez, G. A., Rodríguez Barrero, M. S., \& Moreno Espinosa, M. L. (2018). Inserción social de desmovilizados: Una percepción de la sociedad colombiana. Revista de Ciencias Sociales (RCS), 24(4), 57-69. Recuperado el 2019, de https://dialnet.unirioja.es/servlet/articulo?codigo $=6837100$

Sánchez Blake, E. (2016). La ruta pacífica de las mujeres: repertorios simbólicos en la búsqueda de paz y reconciliación en Colombia. Revista Colombiana de Educación, 1(71), 301-319. doi:10.17227/01203916.71rce301.319

Sepúlveda Romero, M. E., Moreno Martínez, E. P., Tovar Mesa, J. E., Franco Villada, J. A., \& Villarraga Tole, C. L. (2015). Responsabilidad social de las PyMEs en el marco del posconflicto, como soporte para la inclusión laboral de la población reinsertada. Comunicación, Cultura y Política, 45-61. Recuperado el 2019 ,

https://journal.universidadean.edu.co/index.php/revistai/article/view/1335

Unidad para la Atención y la Reparación Integral a las Víctima. (30 de mayo de 2018). Unidad capacita comunidades indígenas de Caquetá sobre rutas de atención. Unidad para la Atención y la Reparación Integral a las Víctima. Obtenido de https://www.unidadvictimas.gov.co/es/asuntos-etnicos/unidadcapacita-comunidades-indigenas-de-caqueta-sobre-rutas-de-atencion/42402 
Unidad para la Atención y la Reparación Integral a las Víctimas. (26 de junio de 2018). Comunidad de El Portal La Mono, en Belén de los Andaquies, recibió caseta comunal. Recuperado el 2019, de https://www.unidadvictimas.gov.co/es/reparacion/comunidad-de-el-portal-lamono-en-belen-de-los-andaquies-recibio-caseta-comunal/42747

Unidad para la Atención y Reparación Integral a las Víctimas. (16 de noviembre de 2018). Aprobado primer plan de retorno étnico en Putumayo. Recuperado el 2019, de https://www.unidadvictimas.gov.co/es/retornos-y-reubicaciones/aprobadoprimer-plan-de-retorno-etnico-en-putumayo/44959

Unidad para la Atención y Reparación Integral a las Víctimas. (09 de Noviembre de 2018). Resguardo de San Pablo del Para avanza en reparación colectiva. Unidad de víctimas. Recuperado el 2019, de https://www.unidadvictimas.gov.co/es/reparacion-colectiva/resguardo-de-sanpablo-del-para-avanza-en-reparacion-colectiva/44862

Vásquez, T. (Abril de 2014). Caquetá análisis de conflictividades. 91. Colombia. Recuperado el 2019, de https://www.undp.org/content/dam/colombia/docs/Paz/undp-co-caqueta2014.pdf

Zwerg Villegas, A. M., \& Jiménez Aguilar, C. M. (2015). Discriminación socioeconómica en la inserción laboral de graduados universitarios: Percepciones de los decanos de facultades de negocios. RMIE, 20(64), 71-93. Recuperado el 2019, de http://www.scielo.org.mx/pdf/rmie/v20n64/v20n64a5.pdf 